\title{
AGN Search From Multicolor Photometric Observations of Faint ROSAT X-ray Sources in a One Square Degree Field
}

\author{
Suijian Xue, Xu Zhou and Haotong Zhang \\ National Astronomical Observatories, Chinese Academy of Sciences. \\ A20 Datun Rd., Chaoyang District, Beijing 100012
}

\begin{abstract}
We report that a volume-limited, X-ray selected AGN sample, including 49 QSO like and starburst galaxies (SBs) at the 0.1-2.4 keV flux level of $\gtrsim 5.3 \times 10^{-14} \mathrm{ergs}^{-2} \mathrm{~s}^{-1}$ was formed with photometric redshifts.
\end{abstract}

\section{Introduction}

75 cataloged X-ray sources from a medium deep ROSAT survey (Molthagen et al. 1997) were found in a 1 sq.deg. field, centered at 09:56:24.46, $+47: 35: 08.4$ (J2000). The field has been heavily CCD imaged with 15 intermediate-band filters covering the wavelength range $3360-9745 \AA$. Full identification of the sample sources will not only extend the results of the ROSAT All-Sky Survey to a fainter limit, but also test recent results of Chandra Deep Surveys in a different larger sky field.

\section{Results}

In total we have made 67 optical identifications from $75 \mathrm{X}$-ray sources with several extragalactic populations. The result is summarized in Table 1., and the procedure of identification illustrated in Figure 1.

Table 1. Identification Summary

\begin{tabular}{lcc}
\hline Population & Numbers & Fraction (\%) \\
\hline All & 75 & 100.0 \\
AGNs & 25 & 33.3 \\
SBs & 24 & 32.0 \\
Galaxies & 9 & 12.0 \\
Stars & 9 & 12.0 \\
Unknown & 8 & 10.7 \\
\hline
\end{tabular}

\section{References}

Molthagen, K., Wendker H.J., \& Briel U.G. 1997, A\&AS, 126, 509 

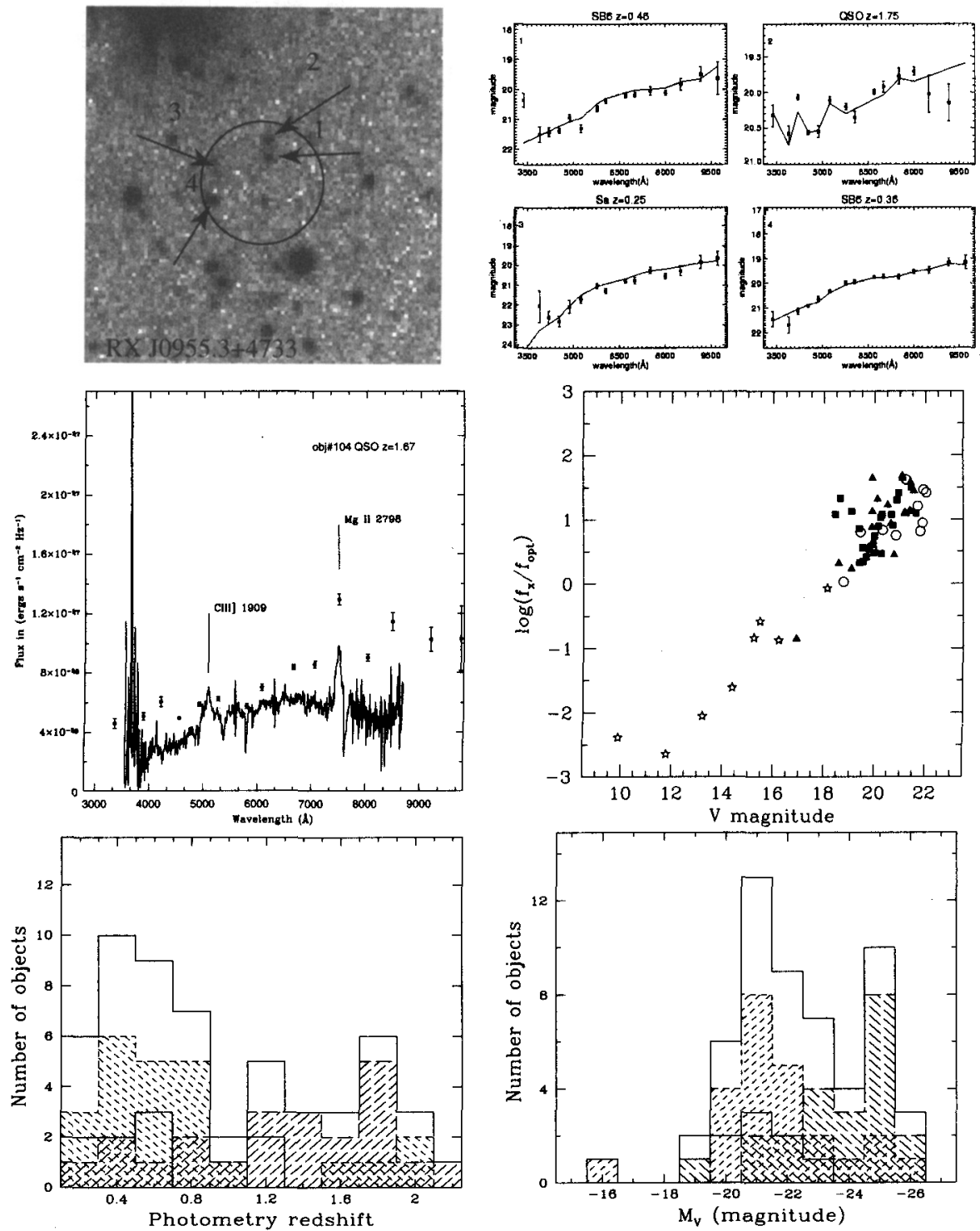

Figure 1. The procedure of source identification shown as an example in top left: 4 optical associations were found within the error circle of RX J0955.3+4733 and their SEDs were measured in 15 colors; top right: 4 sources were classified based on SED fitting with AGN, normal galaxy and star SED templates; middle left: Object 2 was identified with RX J0955.3+4733, and the follow-up spectrum confirms it is a new QSO at $z=1.67$. Middle right: $\mathrm{X}$-ray to optical flux ratio vs. visual magnitude of the sample. AGNs: solid squares; SBs: solid triangles; normal galaxies: circles; stars: stars. Bottom left \& right: the redshift and visual luminosity distributions of identified extragalactic populations. Thick Solid: the total; long dashed: QSOs; short dashed: SBs; thin solid: normal galaxies. 